[1]

${ }^{1}$ Pulmonology, Hospital Beatriz Ângelo, Loures, Portugal.

${ }^{2}$ Cardiothoracic Surgery, Hospital Beatriz Ângelo, Loures, Portugal.

${ }^{3}$ Gynecology and Obstetrics, Hospital Beatriz Ângelo, Loures, Portugal.

${ }^{4}$ Pathology, Hospital Beatriz Ângelo, Loures, Portugal.

\title{
Third time's the charm
}

\section{Case report}

We present the case of a 45-year-old woman who was a smoker (15 pack-years) with no relevant past medical history. She had her menarche at 11 years old with regular cycles and was on oral contraception for $>20$ years.

The patient went to the emergency department complaining of a right-sided pleuritic chest pain of rapid onset and progressive worsening. She had no history of trauma, fever, cough, sputum production or dyspnoea. Physical examination revealed normal blood pressure and heart rate. She was slightly tachypnoeic (respiratory rate of 23 cycles per min) and had a peripheral oxygen saturation of $95 \%$. The right hemithorax was hyper-resonant on percussion and respiratory sounds were absent.

\section{Task 1}

1 Which diagnosis would you consider first?
a. Pneumonia
b. Pleural effusion
c. Pneumothorax
d. Pulmonary embolism

2 Which examination would you request to confirm the diagnosis?
a. Chest radiography
b. Thoracic magnetic resonance
c. Arterial blood gas analysis
d. Chest computed tomography

Cite as: Alvarenga $M$

Clemente S, Calvinho P, et al. Third time's the charm.

Breathe 2017; 13: e72-e78. 


\section{Answer 1 \\ 1 c. Pneumothorax \\ 2 a. Chest radiography}

Chest radiography demonstrated a right pneumothorax with lung collapse (figure 1) and a chest tube was inserted. After lung expansion and chest tube removal, the patient was released from the hospital and stopped smoking.

About a month later, she presented herself again in the emergency department with a similar clinical presentation. Chest radiography confirmed a recurrent right pneumothorax. A chest tube was placed with partial lung expansion. Being a second



Figure 1 Chest radiograph showing a right pneumothorax. episode, a surgical approach through video-assisted thoracic surgery was offered and the patient accepted. A careful inspection of the pleural surfaces and diaphragm was performed, and no lesions were visualised. Small apical blebs were identified, and a superior right lung lobe wedge resection and mechanical pleurodesis (pleural abrasion) were performed. Histopathological examination showed emphysema. The patient was released 5 days after surgery, asymptomatic and with no pneumothorax on the chest radiograph.

1 month later, the patient was observed in a pulmonology consultation. She was asymptomatic but chest radiography showed another recurrent right pneumothorax (figure 2).



Figure 2 Chest radiograph showing a recurrent right pneumothorax.

Task 2

In view of the recurrent nature of the pneumothorax, which other diagnosis would you consider in this patient? 


\section{Answer 2}

Thoracic endometriosis

The patient corroborated that all three pneumothoraces had occurred during the first 2 days of menstruation.

\section{Task 3}

How would you proceed?

a. Observation alone

b. Surgery

c. Chest tube insertion

d. New pulmonology consultation in 3 months with radiography 


\section{Answer 3}

\section{b. Surgery}

No chest tube was inserted and a second video-assisted thoracic surgery was performed. Two small diaphragmatic fenestrations and two


Figure 3 Microscopic examination of reddish lesions identified during thoracic surgery: pleural infiltration by endometrial foci. a) Haematoxylin and eosin (HE), magnifying glass; b) HE, 40x. small reddish lesions were identified. Pleurectomy of the upper half was performed and talc pleurodesis was performed on the lower pleural half. Histopathological examination confirmed endometriosis (figure 3). Oral contraception was stopped and she was given medroxyprogesterone acetate $150 \mathrm{mg}$.

\section{Task 4}

What is the most common site of endometriosis, frequently associated with thoracic endometriosis?
a. Kidneys
b. Pelvic cavity
c. Liver
d. Pericardial cavity 


\section{Answer 4}

b. Pelvic cavity

The patient was later observed on a gynaecology consultation, where dysmenorrhoeal and dyschezial complaints were identified. Gynaecological examination revealed a painful irregular area in the vaginal upper posterior wall suggestive of endometriosis in the rectovaginal septum.

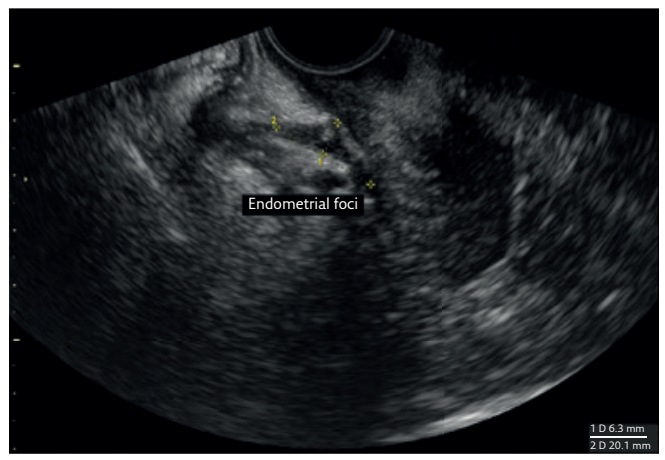

Figure 4 Transvaginal ultrasound showing endometrial foci on rectovaginal septum.
Furthermore, transvaginal ultrasound revealed a fixed uterus with two deep endometrial foci (figure 4). Goserelin $3.6 \mathrm{mg}$ monthly was prescribed, and total hysterectomy with bilateral oophorectomy and removal of rectovaginal endometrial foci were proposed. She had surgery 2 months later with confirmation of endometrial foci on rectovaginal septum (figure 5) and goserelin was suspended. 5 months later, the patient was asymptomatic with no pneumothorax recurrence.



Figure 5 Laparoscopic total hysterectomy with bilateral oophorectomy. 


\section{Discussion}

Catamenial pneumothorax is defined as a spontaneous and recurrent pneumothorax (at least two episodes) that occurs between the day before and $72 \mathrm{~h}$ after the onset of menstruation [1-4]. Some extend it to $72 \mathrm{~h}$ before [4] and others to $96 \mathrm{~h}$ after [5] It represents 3-6\% of spontaneous pneumothoraces in women of childbearing age [4, 6, 7]. In studies evaluating only women referred for thoracic surgery, the proportion was $25-33 \%$ [1, 5, 8].

Endometriosis refers to endometrial tissue outside the uterus, more frequently in the pelvic peritoneum, ovaries and rectovaginal septum. In rare cases, it can occur outside the pelvic cavity, mainly in the thoracic cavity $[9,10]$. The most frequent clinical manifestation is catamenial pneumothorax (72\%) followed by haemoptysis (14\%), haemothorax (12\%) and pulmonary nodules (2\%) [11]. Endometriosis affects $2-10 \%$ of women of childbearing age [9, 12]. Despite being considered rare, the true prevalence of thoracic endometriosis is unknown. Thoracic endometriosis incidence peaks around 35 years of age, 5 years after the peak incidence of pelvic endometriosis [13]. Definitive diagnosis requires identification of characteristic macroscopic lesions (endometrial foci, frequently on the diaphragm, and diaphragmatic defects) and histopathological confirmation. In this case, the diagnosis was only possible after a second thoracic surgery, demonstrating how difficult it can be. In addition, she had pelvic disease that required specific therapy, showing how valuable an interdisciplinary approach is.

The pathophysiology of catamenial pneumothorax is poorly understood. The most accepted theory states that during menstruation, there is no cervical mucus so the peritoneal cavity communicates with exterior through the uterus and Fallopian tubes. Consequently, air can enter pelvic cavity and, through congenital or acquired diaphragmatic defects, it can reach pleural cavity. Being endometriosis characterised by diaphragmatic defects, this theory explains its association with catamenial pneumothorax.

Like catamenial pneumothorax, there are numerous theories that attempt to explain the pathophysiology of thoracic endometriosis. The most accepted one is based on retrograde menstruation. Retrograde menstruation leads to pelvic implantation of endometrial tissue. There is a preferential circulation of fluids/air from right paracolic gutter to right subphrenic space. Here, the liver and the pressure difference between pelvic and thoracic cavities result in migration of fluids/ air from the right subphrenic space to right pleural space, explaining why catamenial pneumothorax is right sided in $85-90 \%$ of cases [4].

There is no consensus on the best therapeutic approach in catamenial pneumothorax, because published data are based on small retrospective studies and case reports [1, 2, 4, 5]. Nevertheless, most experts advocate both medical and surgical approaches in order to minimise recurrences. The thoracic cavity must be carefully inspected and any lesions (endometrial foci, blebs or diaphragmatic defects) resected $[5,8]$, preferably by pleurectomy because it will promote pleurodesis. Chemical basal pleurodesis can also be performed to prevent chronic hiccups. The literature also refers to other techniques such as exclusive chemical pleurodesis with talc [1] or placing an artificial web above the diaphragm whenever diaphragmatic defects are found [2, 5]. Medical therapy aims at endometrial atrophy and amenorrhoea by means of hormonal blockage. Amenorrhoea should be maintained 6-12 months after surgery in order to allow an efficient pleural adhesion [3, 4]. Premature interruption of hormonal therapy can lead to cyclic hormonal changes and pneumothorax recurrence. There are several hormonal therapies: oral contraceptives, danazol, progesterone derivatives and gonadotropin-releasing hormone analogues.

Oral contraceptives have high recurrence rates. This patient had three catamenial pneumothoraces despite taking oral contraceptives for $>20$ years. We hypothesise that oral contraceptives were a protective factor, delaying disease progression and explaining why she is considerably older than we would expect. Although there have been no comparative studies of efficacy, several experts prefer gonadotropinreleasing hormone analogues [2, 4, 5, 13]. However, we have to consider their long-term adverse effects, particularly on bone density, and their significantly higher cost. Accordingly, in this case, a progesterone derivative was chosen as first-line therapy. After deep pelvic endometriosis with possible rectal extension was diagnosed in this multiparous woman not planning another pregnancy, a surgical approach was proposed. Hormonal therapy became a short-term plan (until surgery) and concerns about its long-term use were less important. Therefore, the progesterone derivative was replaced by a gonadotropin-releasing hormone analogue, goserelin.

In conclusion, thoracic endometriosis diagnosis is difficult and requires high clinical suspicion. In women with recurrent pneumothoraces, a temporal relationship with menstruation, rightsided predominance and gynaecological symptoms should alert us to this diagnosis. 


\section{References}

1. Alifano M, Jablonski C, Kadiri H, et al. Catamenial and noncatamenial, endometriosis-related or nonendometriosis-related pneumothorax referred for surgery. Am J Respir Crit Care Med 2007; 176: 1048-1053

2. Leong AC, Coonar AS, Lang-Lazdunski L. Catamenial pneumothorax: surgical repair of the diaphragm and hormone treatment. Ann R Coll Surg Engl 2006; 88: 547-549.

3. Tschopp JM, Bintcliffe O, Astoul P, et al. ERS task force statement: diagnosis and treatment of primary spontaneous pneumothorax. Eur Respir J 2015; 46: 321-335.

4. Visouli AN, Darwiche K, Mpakas A, et al. Catamenial pneumothorax: a rare entity? Report of 5 cases and review of the literature. J Thorac Dis 2012; 4: Suppl. 1, 17-31.

5. Marshall MB, Ahmed Z, Kucharczuk JC, et al. Catamenial pneumothorax: optimal hormonal and surgical management. Eur J Cardiothoracic Surg 2005; 27: 662-666.

6. Shearin RP, Hepper NG, Payne WS. Recurrent spontaneous pneumothorax concurrent with menses. Mayo Clin Proc 1974 49: 98-101.
7. Nakamura H, Konishiike J, Sugamura A, et al. Epidemiology of spontaneous pneumothorax in women. Chest 1986; 89: 378-382.

8. Alifano M, Roth T, Broët SC, et al. Catamenial pneumothorax: a prospective study. Chest 2003; 124: 1004-1008.

9. Giudice LC. Clinical practice. Endometriosis. N EnglJ Med 2010; 362: 2389-2398.

10. Kennedy S, Bergqvist A, Chapron C, et al. ESHRE guideline for the diagnosis and treatment of endometriosis. Hum Reprod 2005; 20: 2698-2704.

11. Channabasavaiah AD, Joseph JV. Thoracic endometriosis: revisiting the association between clinical presentation and thoracic pathology based on thoracoscopic findings in 110 patients. Medicine (Baltimore) 2010; 89: 183-188.

12. Eskenazi B, Warner ML. Epidemiology of endometriosis. Obstet Gynecol Clin North Am 1997; 24: 235-258.

13. Alifano M, Trisolini R, Cancellieri A, et al. Thoracic endometriosis: current knowledge. Ann Thorac Surg 2006; 81: 761-769. 